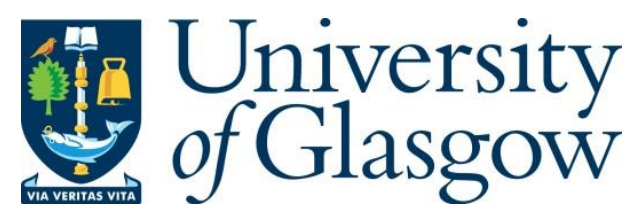

Hadfield, R. H. (2020) Superfast photon counting. Nature Photonics, 14(4), pp. 201-202.

There may be differences between this version and the published version. You are advised to consult the publisher's version if you wish to cite from it.

http://eprints.gla.ac.uk/213251/

Deposited on: 3 April 2020

Enlighten - Research publications by members of the University of Glasgow http://eprints.gla.ac.uk 
Nature Photonics News \& Views March 2020 - prepublication

PHOTODETECTORS

\section{Superfast photon counting}

The news that superconducting nanowire detectors can detect single photons with a timing precision of just a few picoseconds will benefit applications ranging from sensing to quantum communications.

\section{Robert Hadfield}

The ability to detect single photons with high efficiency, precise timing and low noise underpins a host of important applications in photonics, including single-photon LIDAR, fluorescence lifetime measurements, quantum secure communications and experiments in quantum optics. Over the past 20 years, the superconducting nanowire single-photon detector (SNSPD) has become an increasingly popular device for this task. A crucial parameter in the above applications is the SNSPD's timing jitter, the fluctuation between the true arrival time of a photon and the electrically registered arrival time recorded by the system. For example, in quantum communication experiments, the detector timing jitter is typically the limiting factor determining the clock rate of the system. For many years, the minimum timing jitter in SNSPDs has remained at the level of greater than $10 \mathrm{ps}$ and was believed to be limited by the signal to noise ratio of the amplifier chain. In this month's issue of Nature Photonics, Boris Korzh of the NASA Jet Propulsion Laboratory and co-workers report a breakthrough in the area with the demonstration of a superconducting nanowire detector with a timing jitter of below 3 ps full-width at half-maximum [1].

The idea of using a superconducting nanowire for high-speed infrared photon counting dates back almost 20 years to the pioneering work of Gregory Gol'tsman and co-workers at Moscow State Pedagogical University and the University of Rochester [2]. The device concept is striking in its simplicity: a current-carrying superconducting nanowire will temporarily switch from zero to high electrical resistance following the transient thermal hotspot that results from the absorption of a single infrared photon. The resulting output voltage pulse can be readily amplified and registered with room temperature electronics, signalling the arrival of a photon. The nanowire then relaxes swiftly back into the superconducting state as the photon energy is dissipated into the device substrate. Although superconducting detectors of various types have long been prized by astronomers for their excellent sensitivity from X-ray to submillimetre wavelengths [3], the pathway into mainstream photonics for SNSPD technology has been circuitous.

At the time of this first report in 2001 [2], the notion that a nanoscale superconducting device that required liquid helium to operate could play a useful role in photonics applications seemed far-fetched to many optoelectronics experts. However, just a few years later, in 2007, early stage SNSPDs that were integrated into a closed-cycle cooling system set a new world record in long distance quantum cryptography in optical fibre, matching the 40-dB loss threshold required for space-to-ground quantum communications [4]. This feat caught the attention of the wider photonics community; superconducting nanowires began to be considered as a serious alternative to established photon counting solutions such as photomultipliers and single photon avalanche diodes at telecom wavelengths [5].

In 2013, Francesco Marsili and co-workers demonstrated near-unity single-photon detection efficiency with SNSPDs at the key telecom wavelength of $1550 \mathrm{~nm}$ [6]. This near-ideal detector performance enabled a breakthrough loophole-free Bell test of the principles of quantum physics using photons [7]. The absorption enhancement was achieved by integrating a very uniformly fabricated superconducting nanowire device with an appropriate optical cavity and optimising fibre coupling to the microscopic device area. Similar high efficiency SNSPDs have now been commercialised by a growing number of successful start-up companies worldwide and are now a standard photon counting tool in quantum optics labs across the world. Moreover, 
these detector systems are rugged, cost-effective and user-friendly enough for deployment in real-world photoncounting applications.

A tantalizing aspect of the first superconducting nanowire wire device [2] was the projected picosecond timing resolution. This arises due to the device's ultrafast energy relaxation time -- following the absorption of a photon by a single electron in the superconducting material the energy is very rapidly transferred to other electrons forming a region of weakened superconductivity commonly referred to as a hotspot. In practice, this ultimate timing resolution had proven hard to exploit however and in applications timing resolution of $50 \mathrm{ps}$ or greater are typical [8].

This latest study from Korzh et al [1] has definitively addressed this issue, pushing the time resolution of SNSPDs to down below 3 ps. The achievement is largely due to a concerted research effort driven by the Defense Advanced Research Projects Agency (DARPA) Detect program (2016-present) in the US where the country's top experimental groups in this field have worked closely together with strong support from leading theorists. As a result, a precise understanding of the timescales of the detection process in different superconducting materials has been gained including hotspot growth and subsequent breaking of superconductivity.

Korzh et al.'s device success is due to the use of high-quality niobium nitride which is shown to be superior to other superconducting materials such as tungsten silicide or molybdenum silicide. The team also redesigned the detector in order to eliminate geometric-based delay effects associated with photon absorption at different points along an extended nanowire. Finally, good impedance matching to the external circuit and careful choice of cryogenic amplifiers and time-correlated single-photon counting (TCSPC) electronics were employed.

The resulting timing jitter of $2.6 \mathrm{ps}$ in the visible and 4 ps at $1550 \mathrm{~nm}$ with high internal quantum efficiency is remarkable: this is roughly the time it takes a photon to travel just one millimetre in free space. Timing jitter is important as it determines the minimum bin size for time stamping photon arrival events in applications such as fibre optic quantum communication and space-to-ground communications. In remote sensing applications such as single-photon LIDAR or fibre optic sensing, improved timing resolution translates directly to improved spatial resolution for a given acquisition time. The team's superconducting nanowire coupled with suitable TCSPC electronics, could even act as an optical sampling oscilloscope, operating with single photon sensitivity and a bandwidth of over $100 \mathrm{GHz}$ [9].

This important work [1] heralds many exciting future developments and is likely to enable new picosecond photon counting applications in mainstream photonics. Improvements in practical cryogenics are likely to accelerate this trend. Superconducting nanowire single-photon detector device development remains a highly fruitful research area, with academic research groups, national laboratories and start-up companies all engaged. Important avenues of current development include the scale-up from single-pixel devices to large scale arrays [10], integration with complex waveguide circuits [11] and extension of photon counting performance into the ultraviolet and mid-infrared spectral regions.

\section{AUTHOR}

Robert H. Hadfield,

James Watt School of Engineering, University of Glasgow, Glasgow, G12 8QQ, United Kingdom.

E-mail Robert.Hadfield@glasgow.ac.uk

ORCID iD https://orcid.org/0000-0002-8084-4187 


\section{REFERENCES}

[1] Korzh, B. et al. Nature Photonics https://doi.org/10.1038/s41566-020-

0589-x (2020)

[2] Gol'tsman, G. N. et al. Appl. Phys. Lett. 79, 705-707 (2001)

[3] Prober, D. E. Nature 425, 777 (2003)

[4] Takesue, H. et al. Nat. Photon. 1, 343-348 (2007)

[5] Hadfield, R. H. Nat. Photon. 3, 696-705 (2009)

[6] Marsili, F. et al. Nat. Photon. 7, 373 210-214 (2013)

[7] Shalm K.E. et al. Phys. Rev. Lett. 115, 250402 (2015)

[8] Natarajan, C. M. et al. Superconductor Science and Technology 25, 063001 (2012)

[9] Wang X. et al. J. Lightwave Technology 36, 1166-173 (2020)

[10] Wollman, E. E. et al. Opt. Express 27, 35279-35289 (2019)

[11] Pernice, W. H. P. et al. Nat. Commun. 3, 1325 (2012)

[12] Allmaras, J. P. et al. Nano Lett. https://doi.org/10.1021/acs. nanolett.0c00246 (2020).
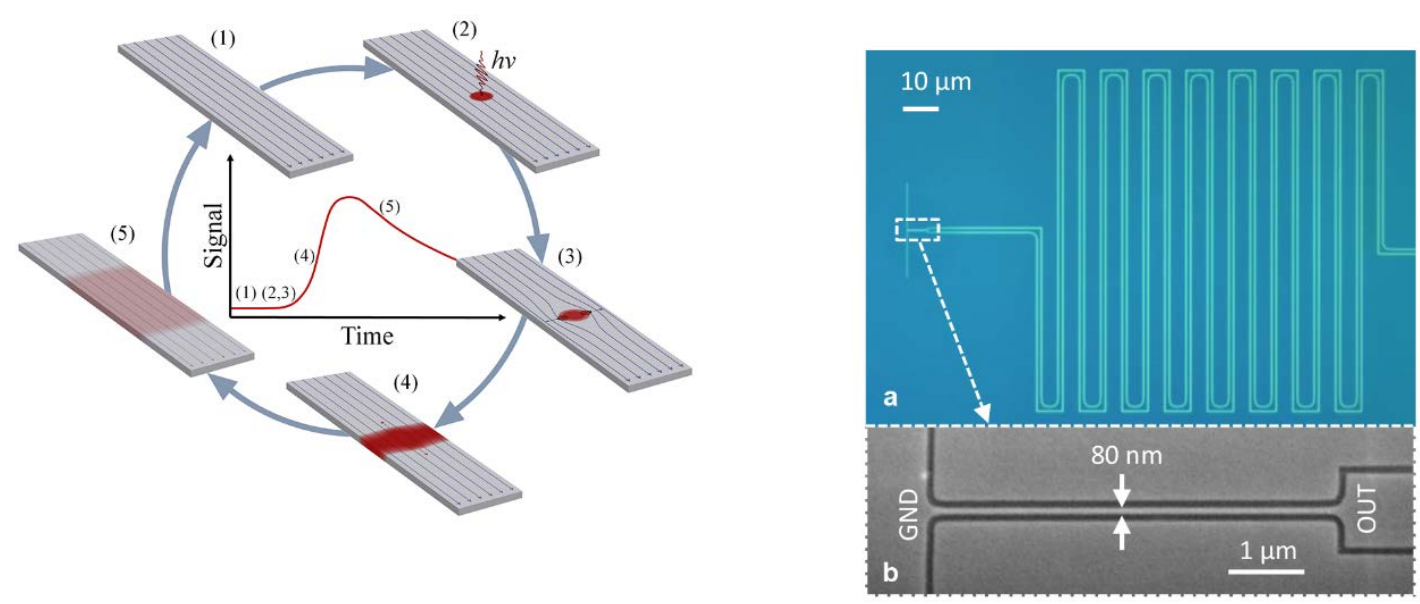

Figure 1 The superconducting nanowire single-photon detector. A - schematic showing the generation of a transient hotspot in a nanowire following the absorption of a single photon (courtesy of Jason P. Allmaras, Ref [12]) (1) supercurrent flows in the nanowire (2) a photon is absorbed by a single electron which relaxes, creating a hotspot of excited quasiparticles (3) Vortex motion breaks superconductivity across the nanowire (4) the normal domain grows along the length of the nanowire leading to a large resistance (5) as current is diverted, the nanowire relaxes back into the superconducting state. B - Microscope images of Korzh et al.'s detector, clearly showing the nanowire's path and geometry. 